\title{
Commentary: Nationwide Surveillance of Novel Oxazolidinone Resistance Gene optrA in Enterococcus Isolates in China from 2004 to 2014
}

\author{
Gianluca Morroni ${ }^{1}$, Andrea Brenciani ${ }^{1 *}$, Serena Simoni ${ }^{1}$, Carla Vignaroli ${ }^{2}$, Marina Mingoia ${ }^{1}$ \\ and Eleonora Giovanetti ${ }^{2}$
}

${ }^{1}$ Departments of Biomedical Sciences and Public Health, Polytechnic University of Marche, Ancona, Italy, ${ }^{2}$ Departments of Life and Environmental Sciences, Polytechnic University of Marche, Ancona, Italy

Keywords: optrA gene, OptrA protein, OptrA variants, enterococci, oxazolidinone resistance

\section{A commentary on}

\section{OPEN ACCESS}

Edited by:

Teresa M. Coque

Instituto Ramón y Cajal de Investigación Sanitaria, Spain

Reviewed by:

Ana R. Freitas,

University of Porto, Portugal

*Correspondence:

Andrea Brenciani

a.brenciani@univpm.it

Specialty section:

This article was submitted to Antimicrobials, Resistance and

Chemotherapy,

a section of the journal

Frontiers in Microbiology

Received: 07 June 2017 Accepted: 11 August 2017 Published: 24 August 2017

Citation:

Morroni G, Brenciani A, Simoni S,

Vignaroli $C$, Mingoia $M$ and Giovanetti E (2017) Commentary:

Nationwide Surveillance of Novel Oxazolidinone Resistance Gene optrA in Enterococcus Isolates in China from

2004 to 2014.

Front. Microbiol. 8:1631.

doi: 10.3389/fmicb.2017.01631
Nationwide Surveillance of Novel Oxazolidinone Resistance Gene optrA in Enterococcus Isolates in China from 2004 to 2014

by Cui, L., Wang, Y., Lv, Y., Wang, S., Song, Y., Li, Y. et al. (2016). Antimicrob. Agents Chemother. 60, 7490-7493. doi: 10.1128/AAC.01256-16

A distictive feature of the novel oxazolidinone-phenicol resistance gene optrA is a variability yielding an encoded OptrA protein-a 655 amino acid sequence-which is variable in turn. The issue of the OptrA variants was more regularly addressed in the early studies of the new resistance than in the following reports. It is thus with particular interest that we read the recent nationwide surveillance study by Cui et al. (2016), where a wide screening of Chinese enterococci for optrA gave the authors an opportunity to repropose the issue of the different variants of the optrA protein.

When optrA was first reported in China from a 1998 to 2014 collection of human and animal enterococci (incidence, 2.0 and $15.9 \%$, respectively), the optrA-carrying plasmid from a human Enterococcus faecalis isolate (E349) was sequenced (accession no. KP399637) (Wang et al., 2015). The relevant optrA-encoded protein is regarded as the wild type, and is hereinafter referred to as OptrA $\mathrm{E}_{349}$. Soon after the discovery of $o p \operatorname{tr} A$, over a thousand enterococci, randomly collected in 2010-2014, were screened for the gene: among the optrA-positive isolates (incidence, 2.9\%), nine different variants of the OptrA sequence (one being identical to OptrA $\mathrm{E}_{449}$ ) were detected (Cai et al., 2015). Seventeen optrA-positive, unrelated isolates of E. faecalis from the aforementioned 19982014 collection disclosed optrA sequences consistent with no new OptrA variant (seven isolates had OptrA $\mathrm{E}_{\mathrm{E} 49}$ ) (He et al., 2016). Finally—in China, yet again-while screening over two thousand enterococci collected in 2004-2014, Cui et al. (2016) detected among the optrA-positive isolates (incidence, 2.0\%) three new OptrA variants. Thus, the different OptrA sequences so far described in Chinese enterococci total 12 (including OptrA $\mathrm{E}_{349}$ ).

Meanwhile, as soon as the optrA sequence became available, we detected in Italy the gene-first report of optrA outside China-in two clinically distinct but virtually identical Enterococcus faecium isolates from a collection of 81 blood enterococci (incidence, 2.5\%) recovered in 2015 (Brenciani et al., 2016). One of the two E. faecium isolates (strain E35048) was investigated for molecular traits, and its optrA gene (accession no. KT892063) displayed 98\% DNA identity to the wild type gene. 
TABLE 1 | Enterococcus isolates (plus one Staphylococcus isolate) where 14 different OptrA sequences (the E. faecalis wild type, 12 enterococcal variants, and 1 S. sciuri variant) have so far been documented.

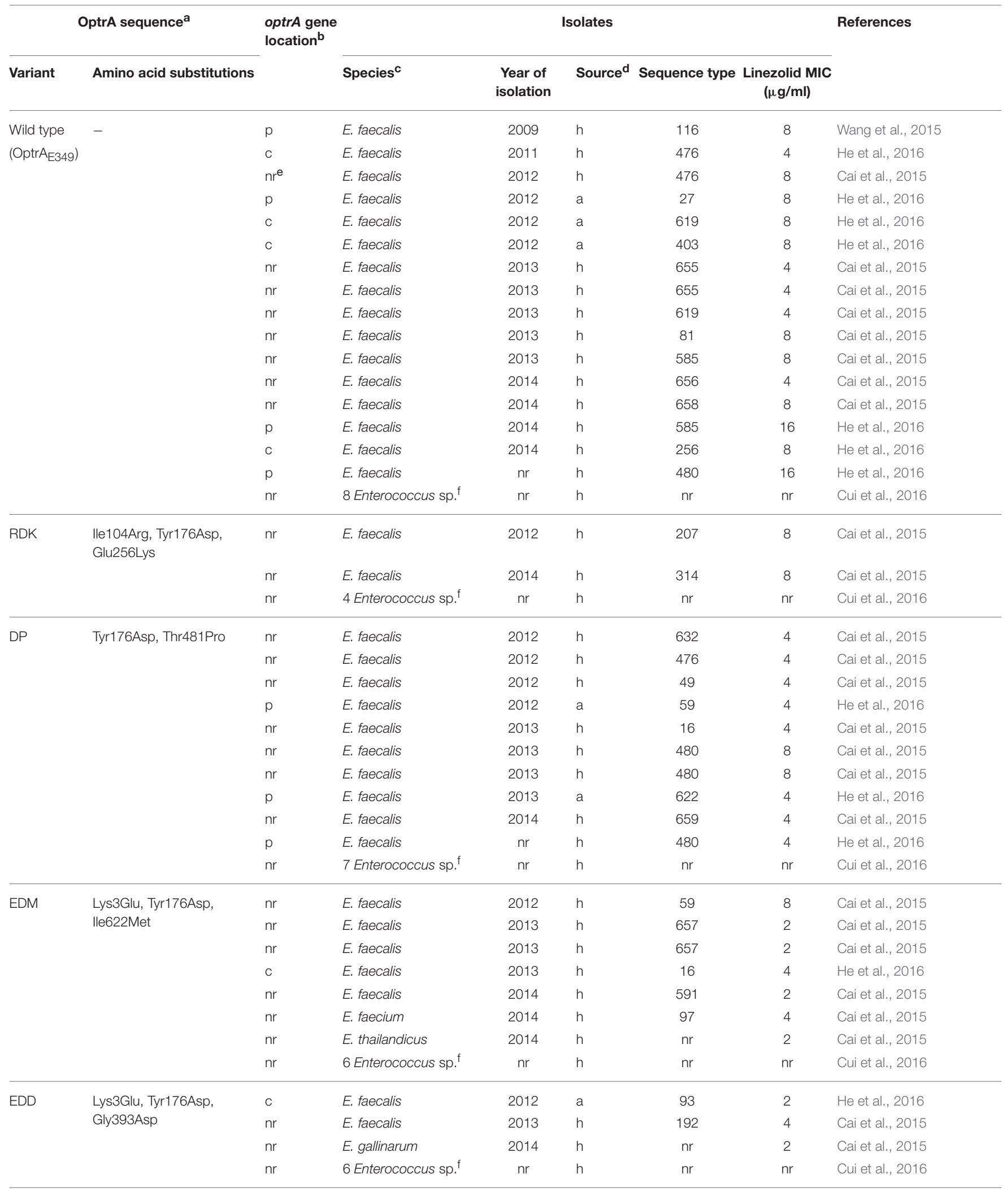


TABLE 1 | Continued

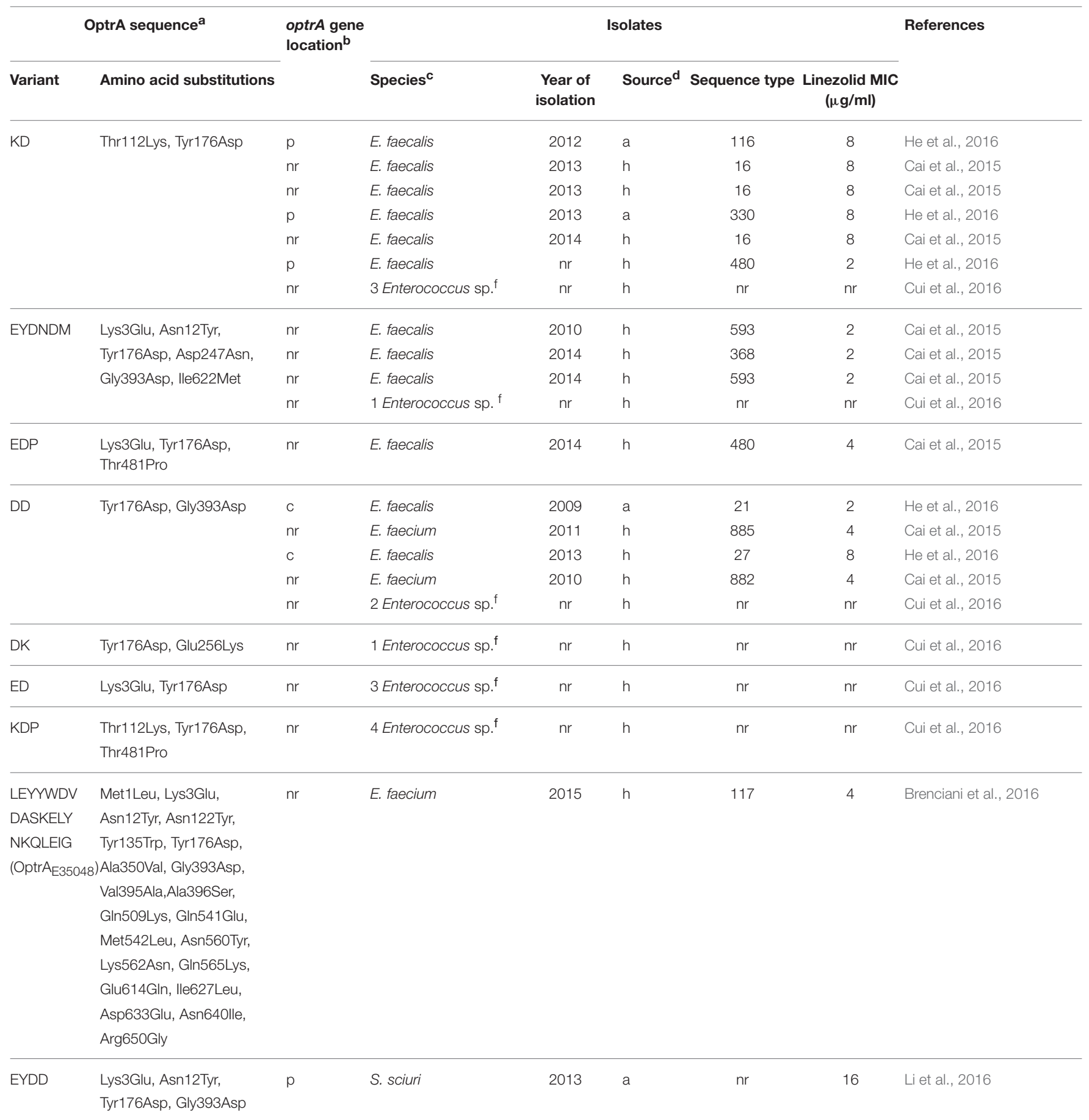

a Variant: the substituting amino acids are given using the single-letter code. Substitutions: amino acid substitutions and their positions.

boptrA location: p, plasmid; c, chromosome.

${ }^{c}$ All species reported in this column are Enterococcus species, except for the one reported on the last line which is a Staphylococcus species (S. sciuri).

'Source: h, human; a, animal.

${ }^{e} n r$, not reported.

${ }^{f}$ Enterococcus species not specified.

In the light of the later data about the diversity of OptrA variants detected in China, it's apparent that our variant (hereinafter referred to as $\mathrm{Optr}_{\mathrm{E} 35048}$ ) is much more dissimilar from OptrA $\mathrm{E}_{\mathrm{E} 39}$ than Chinese variants. Altogether, the reported Chinese variants differ from $\operatorname{Optr}_{\mathrm{E} 349}$ for two, three, or six amino acid substitutions, whereas OptrA $\mathrm{E}_{\mathrm{E} 35048}$ differs from 
OptrA $A_{\mathrm{E} 349}$ for 21 substitutions, 17 of which (i.e., except those at positions 3, 12, 176, and 393) undetected in Chinese isolates. OptrA $\mathrm{E}_{\mathrm{E} 35048}$ adds thus as a more distant variant to the OptrA variants detected in Chinese enterococci. OptrA $A_{\mathrm{E} 349}$ and the currently available enterococcal OptrA variants are summarized in Table 1 together with a number of relevant properties (the optrA gene location and the species, year of isolation, source, sequence type and linezolid MIC of individual isolates, whenever available). In particular, the frequent location of the optrA gene on conjugative plasmids makes the OptrA-mediated linezolid resistance transferable, an obvious cause for concern in view of possible resistance spread (Wang et al., 2015; He et al., 2016).

While recently investigating three optrA-positive E. faecalis isolates of poultry origin in Colombia, Cavaco et al. (2017) deduced that two carried an optrA gene identical to one already detected in China, whereas the third isolate bore an optrA gene with a different nucleotide sequence that was defined as "more closely related" to the one we had described in Italy.

Worryingly, the optrA gene has been found in China not only in enterococci, but also in staphylococci, specifically in a Staphylococcus sciuri strain of swine origin (Li et al., 2016): optrA and its promoter region exhibited 99.1\% nucleotide sequence identity to the corresponding region on the wild type E. faecalis plasmid pE349. The 655 amino acid OptrA sequence from $S$. sciuri is another variant exhibiting $99.4 \%$ identity to OptrA $A_{\mathrm{E} 349}$ (Table 1).

It's self-evident that optrA is not a conserved gene. The related variability of OptrA proteins appears to be a fitting example of that evolvability of clinical resistance by the antibiotic's effect which has been the subject of a recent reflection by Baquero et al. (2013). Given the importance of oxazolidinones as last resort antibiotics for the treatment of serious infections caused by Gram-positive pathogens, it would be important to clarify how the different amino acid substitutions affect OptrA-mediated resistance. However, irrespective of the variant, the linezolid MICs for the optrApositive enterococci listed in Table 1 display limited variability $(2-16 \mu \mathrm{g} / \mathrm{ml})$, the highest MIC value in the range being shared by the optrA-positive strain of S. sciuri. Remarkably,

\section{REFERENCES}

Baquero, F., Tedim, A. P., and Coque, T. M. (2013). Antibiotic resistance shaping multi-level population biology of bacteria. Front. Microbiol. 4:15. doi: $10.3389 /$ fmicb.2013.00015

Brenciani, A., Morroni, G., Vincenzi, C., Manso, E., Mingoia, M., Giovanetti, E., et al. (2016). Detection in Italy of two clinical Enterococcus faecium isolates carrying both the oxazolidinone and phenicol resistance gene optrA and a silent multiresistance gene cfr. J. Antimicrob. Chemother. 71, 1118-1119. doi: $10.1093 / \mathrm{jac} / \mathrm{dkv} 438$

Cai, J., Wang, Y., Schwarz, S., Lv, H., Li, Y., Liao, K., et al. (2015). Enterococcal isolates carrying the novel oxazolidinone resistance gene optrA from hospitals in Zhejiang, Guangdong, and Henan, China, 2010-2014. Clin. Microbiol. Infect. 21, 1095.e1-1095.e4. doi: 10.1016/j.cmi.2015.08.007

Cavaco, L. M., Bernal, J. M., Zankari, E., Léon, M., Hendriksen, R. S., PerezGutierrez, E., et al. (2017). Detection of linezolid resistance due to the optrA gene in Enterococcus faecalis from poultry meat from the American our optrA-positive E. faecium (Brenciani et al., 2016), in spite of no less than 21 amino acid substitutions, exhibits the same linezolid MIC $(4 \mu \mathrm{g} / \mathrm{ml})$ as several Chinese isolates with other OptrA variants, suggesting that the number of amino acid substitutions has little influence on the level of linezolid resistance.

On the other hand, the linezolid resistance breakpoint is still an unsettled issue: indeed, an enterococcus with a linezolid MIC of $4 \mu \mathrm{g} / \mathrm{ml}$ is regarded as "intermediate" according to Clinical Laboratory Standards Institute (2017) and "susceptible" according to European Committee on Antimicrobial Susceptibility Testing (2017). The latter Committee, in particular, sets for enterococci a linezolid epidemiological cut-off of $4 \mu \mathrm{g} / \mathrm{ml}$, and has increased the susceptible clinical breakpoint of linezolid to $\leq 4 \mu \mathrm{g} / \mathrm{ml}$ to avoid dividing wild type MIC distributions (European Committee on Antimicrobial Susceptibility Testing, 2017). In spite of the low linezolid MICs for several optrA-positive isolates, it's well established that in the clinical setting, as well as with other antibiotics, resistance levels may increase in patients with risk factors such as previous linezolid therapy, prolonged exposure to linezolid, and intensive care unit stay (Endimiani et al., 2011; Mendes et al., 2014).

In conclusion, we share and support many recent studies recommending routine surveillance of enterococci for the presence of the optrA gene. In addition, however, we wish for a more extensive interest in the OptrA variants and their correlation with oxazolidinone and phenicol MICs.

\section{AUTHOR CONTRIBUTIONS}

All authors listed have made a substantial, direct and intellectual contribution to the work, and approved it for publication.

\section{ACKNOWLEDGMENTS}

We are grateful to Pietro E. Varaldo for constructive discussion and critical reading of the manuscript. continent (Colombia). J. Antimicrob. Chemother. 72, 678-683. doi: 10.1093/jac/ dkw490

Clinical and Laboratory Standards Institute (2017). M100-S27. Performance Standards for Antimicrobial Susceptibility Testing; Twenty-Seventh Informational Supplement, Wayne, PA: Clinical and Laboratory Standards Institute.

Cui, L., Wang, Y., Lv, Y., Wang, S., Song, Y., Li, Y., et al. (2016). Nationwide surveillance of novel oxazolidinone resistance gene optrA in Enterococcus isolates in China from 2004 to 2014. Antimicrob. Agents Chemother. 60, 7490-7493. doi: 10.1128/AAC.01256-16

Endimiani, A., Blackford, M., Dasenbrook, E. C., Reed, M. D., Bajaksouszian, S., Hujer, A. M., et al. (2011). Emergence of linezolid-resistant Staphylococcus aureus after prolonged treatment of cystic fibrosis patients in Cleveland, Ohio. Antimicrob. Agents Chemother. 55, 1684-1692. doi: 10.1128/AAC.01 308-10

European Committee on Antimicrobial Susceptibility Testing (2017). Available online at: http://www.eucast.org/ 
He, T., Shen, Y., Schwarz, S., Cai, J., Lv, Y., Li, J., et al. (2016). Genetic environment of the transferable oxazolidinone/phenicol resistance gene optrA in Enterococcus faecalis isolates of human and animal origin. J. Antimicrob. Chemother. 71, 1466-1473. doi: 10.1093/jac/dkw016

Li, D., Wang, Y., Schwarz, S., Cai, J., Fan, R., Li, J., et al. (2016). Co-location of the oxazolidinone resistance genes optrA and $c f r$ on a multiresistance plasmid from Staphylococcus sciuri. J. Antimicrob. Chemother. 71, 1474-1478. doi: $10.1093 / \mathrm{jac} / \mathrm{dkw} 040$

Mendes, R. E., Deshpande, L. M., and Jones, R. N. (2014). Linezolid update: stable in vitro activity following more than a decade of clinical use and summary of associated resistance mechanisms. Drug Resist. Updat. 17, 1-12. doi: 10.1016/j.drup.2014.04.002

Wang, Y., Lv, Y., Cai, J., Schwarz, S., Cui, L., Hu, Z., et al. (2015). A novel gene, optrA, that confers transferable resistance to oxazolidinones and phenicols and its presence in Enterococcus faecalis and Enterococcus faecium of human and animal origin. J. Antimicrob. Chemother. 70, 2182-2190. doi: $10.1093 / \mathrm{jac} / \mathrm{dkv1} 16$

Conflict of Interest Statement: The authors declare that the research was conducted in the absence of any commercial or financial relationships that could be construed as a potential conflict of interest.

Copyright (C) 2017 Morroni, Brenciani, Simoni, Vignaroli, Mingoia and Giovanetti. This is an open-access article distributed under the terms of the Creative Commons Attribution License (CC BY). The use, distribution or reproduction in other forums is permitted, provided the original author(s) or licensor are credited and that the original publication in this journal is cited, in accordance with accepted academic practice. No use, distribution or reproduction is permitted which does not comply with these terms. 